\title{
Methyl lucidone exhibits neuroprotective effects on glutamate-induced oxidative stress in HT-22 cells via Nrf-2/HO-1 signaling
}

Jee-Yun Park ${ }^{1,4 \dagger}$, Khulan Amarsanaa ${ }^{1 \dagger}$, Yanji Cui ${ }^{1,6}$, Ji-Hyung Lee ${ }^{1}$, Jinji Wu ${ }^{1}$, Yoon-Sil Yang ${ }^{1,5}$, Su-Yong Eun ${ }^{1,2}$ and Sung-Cherl Jung $1,2,3^{*}$ (D)

\begin{abstract}
Oxidative stress causes neuronal cell death in various neurodegenerative diseases, such as Alzheimer's disease, ischemia, and Parkinson's disease. Therefore, reducing intracellular reactive oxygen species (ROS) has been evaluated as an effective treatment strategy for neurodegenerative disorders. Methyl lucidone (MLC) extracted from Lindera erythrocarpa Makino (Lauraceae) has been previously reported to exhibit microglial-mediated neuroprotective effects via inhibiting neuroinflammation. However, the antioxidant effects of MLC are still unclear. The aim of this study was to determine the neuroprotective mechanism of MLC in HT-22 neurons against oxidative stress induced by glutamate. In results, the pretreatment of MLC significantly enhanced the viability of HT-22 cells under glutamate-induced oxidative conditions, suggesting that MLC has a neuronal mechanism to protect neurons without microglial regulation. Also, the glutamate effect to increase ROS production was effectively blocked by MLC without any free radical scavenging activity. To induce this antioxidant effect, MLC upregulated the expression of heme oxygenase 1 (HO-1) and nuclear translocation of nuclear factor-E2-related factor 2 (Nrf-2), known as an intracellular antioxidant enzyme, and its transcription factor. Additionally, Akt phosphorylation regulating Nrf-2 was confirmed to be involved in the neuroprotective signaling activated by MLC. These results indicate that MLC may play a role as an antioxidant agent to inhibit neurodegenerative processes via activating antioxidant signaling pathways that include Nrf-2 and phosphatidylinositol 3-kinase (PI3K).
\end{abstract}

Keywords: Methyl lucidone, HO-1, Nrf-2, ROS, Neuroprotection, PI3K, Antioxidant

\section{Introduction}

Neurotoxicity in glutamatergic neurons is the major cause to induce cell death in neurodegenerative diseases, such as Alzheimer's disease, ischemia, and Parkinson's disease [1-3]. Excessive extracellular glutamate in the central nervous system (CNS) lowers glutathione levels, inhibiting cystine/glutamate transporters, which cause the accumulation of reactive oxygen species (ROS) and neuronal cell death. Thus, it is called oxidative glutamate toxicity [4].

\footnotetext{
*Correspondence: jungsc@jejunu.ac.kr

${ }^{\dagger}$ Jee-Yun Park and Khulan Amarsanaa equally contributed to this study ${ }^{1}$ Department of Physiology, School of Medicine, Jeju National University, 102 Jejudaehakno, Jeju-si, Jeju-do 63243, Republic of Korea Full list of author information is available at the end of the article
}

To moderate oxidative damage and maintain cellular redox homeostasis, many types of mammalian cells possess a variety of antioxidant systems [5]. One of the important intracellular enzymes for antioxidant signaling is heme oxygenase 1 (HO-1). Nuclear factor-E2-related factor-2 (Nrf-2) regulates the expression of genes encoding phase II detoxification enzymes and antioxidants, including HO-1 [6]. Because Nrf-2 is negatively regulated by Kelch-like ECH-associated protein1 (Keap1) and Nrf-2 phosphorylation is required for its dissociation from Keap1, various kinases, such as phosphatidylinositol 3-kinase (PI3K), also participate in the HO-1-mediated antioxidant signaling pathway $[7,8]$.

Lindera erythrocarpa Makino (Lauraceae), a deciduous shrub, is widely distributed in Korea, Taiwan, Japan, and 
China. The fruit of L. erythrocarpa is used in traditional medicine as an analgesic, antibacterial agent, antidote, digestive, and diuretic agent [9]. The extract of the fruit includes four cyclopentenediones, linderone, lucidone, methyl linderone and methyl lucidone (MLC). Of them, lucidone has been reported to inhibit human farnesyl protein transferase (FPTase) activity and lucidone and MLC effectively suppressed NO production [10]. We also reported that MLC had a neuroprotective effect via inhibiting microglia-mediated neurotoxicity [11]. However, it is still unclear if MLC contributes to the activation of antioxidant signaling in neurons without microglial involvement. To investigate this, we tested the effect of MLC on the HO-1-mediated antioxidant signaling pathway in HT-22 cells.

The results showed that MLC preserved the survival of HT-22 cells under glutamate-induced oxidative conditions via activating the $\mathrm{HO}-1$ antioxidant enzyme and that the activation of PI3K/Akt signaling was required for the antioxidant effect. Our findings in this study suggest that MLC may be a potent antioxidant agent to protect neurons and inhibit the progression of neurodegenerative diseases.

\section{Materials and methods Reagents}

Dulbecco's modified Eagle's medium (DMEM), fetal bovine serum (FBS) and penicillin/streptomycin were purchased from Gibco BRL (Grand Island, NY, USA). The antibody against phospho-Akt was purchased from Cell Signaling Technology (Danvers, MA, USA). Antibodies against Akt and $\mathrm{Nrf}-2$ were purchased from Santa Cruz Biotechnology Inc. (Santa Cruz, CA, USA). The antibodies against heme oxygenase-1 (HO-1) and TATA binding protein (TBP) were purchased from Millipore (Temecula, CA, USA) and Abcam (Cambridge, UK), respectively. The antibody against $\beta$-actin was purchased from SigmaAldrich (St. Louis, MO, USA) and 3-(4,5-dimethylthiazol-2-yl)-2,5-diphenyl tetrazolium bromide (MTT) from Amresco (Solon, OH, USA). 2',7'-Dichlorofluorescin diacetate (DCF-DA) and 2,2-diphenyl-1-picrylhydrazyl (DPPH) were purchased from Sigma-Aldrich (St. Louis, MO, USA). LY294002 and Tin protoporphyrin IX dichloride (SnPP) were purchased from Tocris Bioscience (Bristol, UK). MLC was given by Dr. BM Kwon (Korea Research Institute of Bioscience and Biotechnology, South Korea).

\section{Cell culture}

HT-22 neurons, an immortalized hippocampal neuronal cell line [12], were a generous gift from Dr. B. H. Lee (Gachon University of Medicine and Science, South Korea). The cells were cultured in DMEM supplemented with $10 \% \mathrm{FBS}$ and $1 \%$ penicillin/streptomycin, and incubated at $37{ }^{\circ} \mathrm{C}$ under $5 \% \mathrm{CO}_{2}$.

\section{Measurement of cell viability}

Neuronal viability of HT-22 cells was determined by hiring MTT assay. HT-22 cells were first seeded at a density of $5 \times 10^{4}$ cells/well in a 24 -well plate. After $12 \mathrm{~h}$, cells were treated with MLC at various concentrations for $1 \mathrm{~h}$ and then washed with DMEM. Next, the cells were again treated with $5 \mathrm{mM}$ glutamate for $12 \mathrm{~h}$. For MTT measurement, $100 \mu \mathrm{l}$ of MTT solution $(2 \mathrm{mg} / \mathrm{ml})$ was added to each well and cells were incubated again at $37^{\circ} \mathrm{C}$ for $2 \mathrm{~h}$. After the incubation, the culture supernatant was removed and the MTT formazan crystals were dissolved with $300 \mu \mathrm{l}$ of DMSO. The absorbance was measured at $550 \mathrm{~nm}$ wave length using a microplate reader (Model 550, Bio-Rad, USA).

\section{Measurement of intracellular ROS level}

The intracellular ROS level was measured by using DCFDA. HT-22 cells were seeded at a density of $5 \times 10^{4}$ cells/ well in a 24-well plate and incubated for $12 \mathrm{~h}$. The cells were treated with MLC at several non-toxic concentrations for $1 \mathrm{~h}$ and then washed with DMEM. Next the cells were again treated with $5 \mathrm{mM}$ glutamate for $12 \mathrm{~h}$. For ROS measurement, the cells were loaded with $50 \mu \mathrm{M}$ DCF-DA for $15 \mathrm{~min}$. The fluorescence intensity was detected by using a fluorescence reader (Spectra Fluor, Tecan, Austria) at an excitation wavelength of $485 \mathrm{~nm}$ and an emission wavelength of $535 \mathrm{~nm}$.

\section{Measurement of free radical scavenging effect}

$\mathrm{DPPH}$ assay was performed to measure a free radical scavenging activity of MLC. MLC and N-acetyl cysteine (NAC) $(10 \mu \mathrm{l})$ were added to $190 \mu \mathrm{l} \mathrm{DPPH}(0.15 \mathrm{mM})$ in each well (96-well plate) and mixed vigorously. The mixture was incubated at room temperature for $1 \mathrm{~h}$ in the dark by covering with aluminum foil. The absorbance was detected at $517 \mathrm{~nm}$ wavelength using a microplate reader (VersaMax, Molecular devices, USA).

\section{Preparation of cytoplasmic and nuclear protein}

The preparation of cytoplasmic and nuclear proteins was performed by using NE-PER Nuclear and Cytoplasmic Extraction Reagents (Invitrogen, USA) according to manufacturer's protocols. HT-22 cells were seeded at a density of $1 \times 10^{6}$ cells/dish in $100 \mathrm{~mm}$ dish. The cells were treated with MLC during the time indicated in results. Then, they were washed twice and collected with cold phosphate-buffered saline (PBS; pH 7.4). After centrifuging, cell pellets were resuspended in a Cytoplasmic extraction reagent. After centrifuging them again, the supernatant cytoplasmic extract was transferred to a new 
tube. And then, the nuclear pellets were resuspended in a nuclear extraction reagent, and centrifuged. The supernatant nuclear protein extract was transferred to a new tube and stored at $-80^{\circ} \mathrm{C}$ until using them.

\section{Western blot analysis}

Cell extracts were separated with 12\% SDS-PAGE and transferred to a polyvinylidene difluoride (PVDF) membrane (Bio-Rad Laboratories, CA, USA). After blocking with 5\% skim milk in TBS (25 mM Tris, pH 7.4, $150 \mathrm{mM}$ $\mathrm{NaCl})$, membranes were probed with anti-Nrf-2 (1:200), anti-HO-1 (1:1000), anti-phospho-Akt (1:1000), anti-Akt (1:200), anti- $\beta$-actin $(1: 5000)$ or anti-TBP $(1: 1000)$. Blots were washed three times with TBS including $1 \%$ tween20 and incubated with HRP-conjugated anti-mouse or anti-rabbit antibody. Then the blots were detected using an enhanced chemiluminescence reagent according to the manufacturer's protocol. Optical densities of the band were quantified by using an Image J program.

\section{Statistics analysis}

The data were presented as the mean value \pm SEM through at least 3 independent experiments. Statistical analysis was performed using Student $t$ test and oneway ANOVA. The differences between groups were considered to be statistically significant when $p<0.05$ or $p<0.01$.

\section{Results}

MLC increases neuronal viability under glutamate-induced oxidative condition

To investigate whether MLC protected neurons against oxidative cell death, we observed the cytotoxic effects of glutamate $(5 \mathrm{mM})$ on HT-22 cells with or without
MLC pretreatment. In cytotoxicity tests conducted to determine the nontoxic MLC concentrations, MLC was not cytotoxic at concentrations less than $5 \mu \mathrm{M}$ (Fig. 1a). Figure $1 \mathrm{~b}$ shows that treatment with $5 \mathrm{mM}$ glutamate for $12 \mathrm{~h}$ significantly reduced the viability of HT-22 cells compared to non-treated cells (MLC $0 \mu \mathrm{M}$; - Glu: $100.0 \pm 1.4,+$ Glu: $24.7 \pm 0.7 \%, p<0.01)$. In contrast, cells pretreated with MLC $(0.5-5 \mu \mathrm{M})$ showed significantly higher viability in a dose-dependent manner than that of glutamate-treated cells without MLC pretreatment (+Glu; $30.3 \pm 0.7,35.7 \pm 0.4,63.3 \pm 0.4$, and $83.6 \pm 1.1 \%$ at $0.5,1,3$, and $5 \mu \mathrm{M}$ MLC, respectively). These results clearly demonstrate that MLC protected HT-22 cells against glutamate-induced cytotoxicity. As the results in Fig. 1 show, all other experiments were performed at or lower than $5 \mu \mathrm{M} \mathrm{MLC}$, showing maximal neuroprotective effects without cytotoxicity.

\section{MLC inhibits glutamate-induced ROS production in HT-22 neurons}

Excessive extracellular glutamate is known to cause neuronal damage by inducing oxidative toxicity and inhibiting ROS production blocks neuronal damage under glutamate-induced oxidative conditions $[4,13]$. Because MLC significantly blocked glutamate-induced neuronal death (Fig. 1b), it was necessary to determine whether MLC suppressed the glutamate-induced ROS production in HT-22 cells. The results of the DCF-DA assay showed that $5 \mathrm{mM}$ glutamate significantly increased ROS levels up to $133.9 \pm 4.8 \%(0 \mu \mathrm{M}$ MLC, + Glu $)$. However, in cells pretreated with MLC, the glutamate-induced ROS was markedly reduced to control level (Fig. 2a, $5 \mu \mathrm{M}$ MLC, +Glu: $107.0 \pm 4.7 \%, \quad p<0.01$ compared to MLC $0 \mu \mathrm{M},+\mathrm{Glu})$. This provides evidence that the
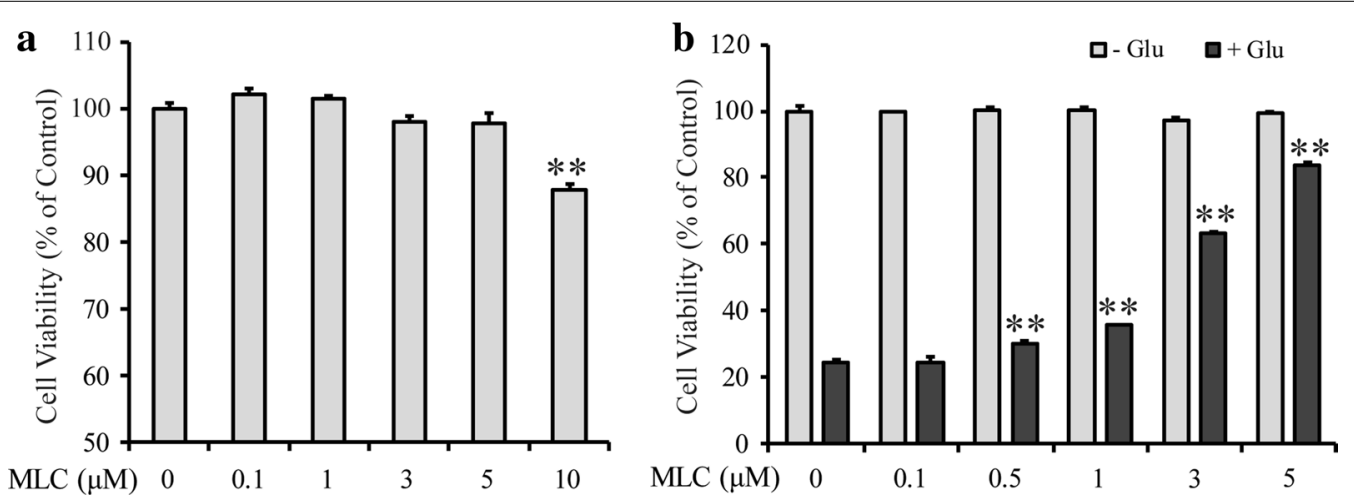

Fig. 1 MLC protects HT-22 neurons against glutamate-induced neuronal cell death. a HT-22 cells were treated for $1 \mathrm{~h}$ with various concentrations of MLC to observe its own cytotoxicity. After washing out, the cells were incubated for $12 \mathrm{~h}$ in fresh media without MLC. All measurements of cell viability in this study were examined using MTT assay. ${ }^{*} p<0.01$, compared to cells not treated with MLC $(0 \mu \mathrm{M})$. $\mathbf{b}$ After pretreating with MLC for $1 \mathrm{~h}$, the cells were washed and further incubated with glutamate $(5 \mathrm{mM})$ for $12 \mathrm{~h} .{ }^{* *} p<0.01$, compared to the group pretreated with MLC alone. The data indicate mean \pm SEM $(n=3)$ 

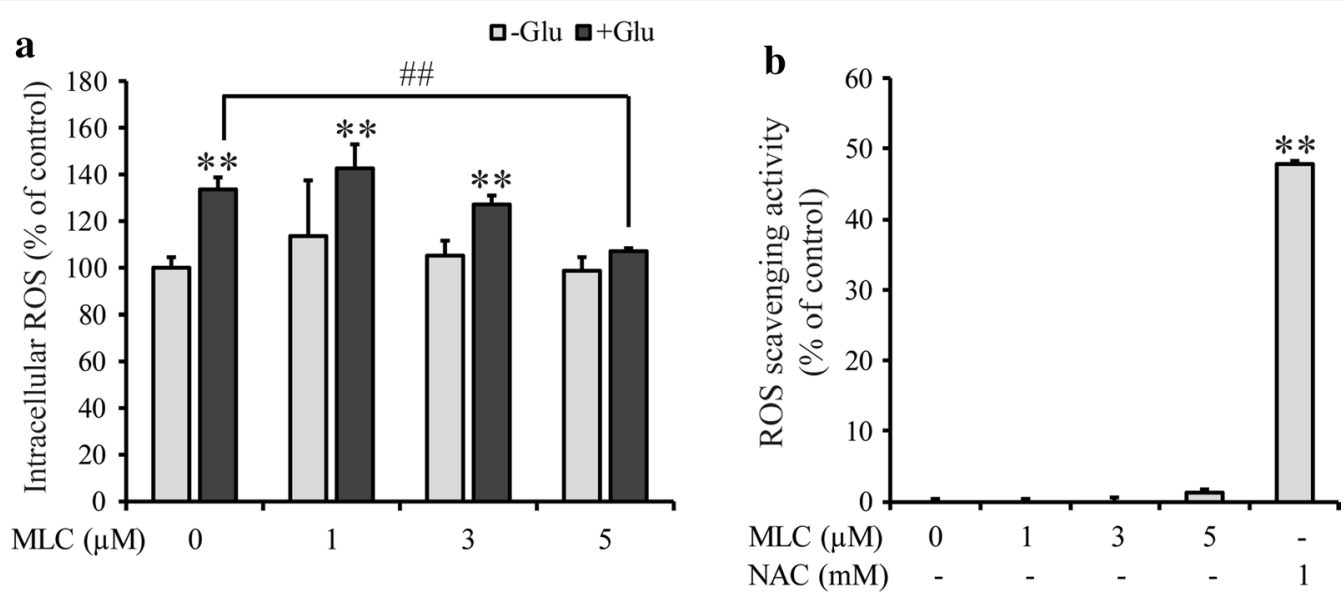

Fig. 2 MLC suppresses glutamate-induced ROS production in HT-22 cells. a After pretreating with MLC $(1,3,5 \mu M)$ for $1 \mathrm{~h}$, the cells were washed and further incubated with glutamate $(5 \mathrm{mM})$ for $12 \mathrm{~h}$ in the absence of MLC. Intracellular ROS levels were measured using ROS-sensitive fluorescent DCF-DA. ${ }^{* *} p<0.01$, compared to cells not treated with any drugs $(0 \mu \mathrm{M} \mathrm{MLC}$ and $-\mathrm{Glu})$, and ${ }^{\# \#} p<0.01$, compared to the group treated with glutamate alone at $0 \mu \mathrm{M} \mathrm{MLC}$. $\mathbf{b}$ DPPH assay was performed to measure the antioxidative effect of MLC and the rate of free radical scavenging activities was calculated as inhibition rate (\%). ${ }^{* *} p<0.01$, compared to cells not treated any drugs. The data indicate $m e a n \pm S E M(n=3)$

MLC-increased viability of HT-22 cells may be due to the inhibition of glutamate-induced ROS production. Next, the DPPH assay was performed to know if the antioxidant ability of MLC involved ROS scavenging effects by using antioxidant $N$-acetyl cysteine (NAC) as a positive control. In this experiment, MLC did not show scavenging effects, unlike NAC exhibiting ROS scavenging activity (Fig. $2 \mathrm{~b}, 5 \mu \mathrm{M}$ MLC: $1.2 \pm 0.5 \%$; NAC: $47.8 \pm 0.3 \%$, $p<0.01)$. These results indicate that MLC may play a role to reduce glutamate-induced ROS production by regulating the intracellular pools of ROS scavengers.

\section{MLC activates Nrf-2/HO-1 signaling transduction}

In Fig. 2, the fact that MLC reduced intracellular ROS without scavenging effects strongly suggests a possibility that MLC influences the cellular regulation of antioxidant enzymes. Because HO-1 catalyzing oxidative degradation contributes to the reduction of oxidative toxicity and increases the neuronal survival rate, we hired Western blot analysis to determine if MLC might influence the expression of HO-1 [14, 15]. In Fig. 3a, a significant induction of HO-1 was observed since $3 \mathrm{~h}$ after MLC treatment, showing a maximal level at $6 \mathrm{~h}(100.0 \pm 13.9$,

\section{a}
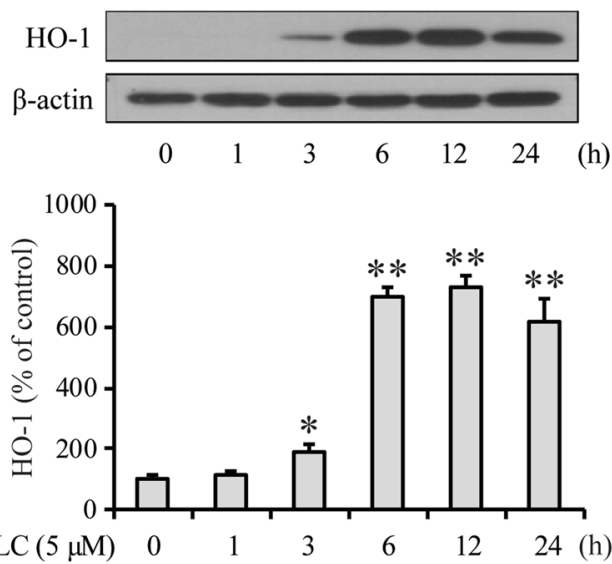

b
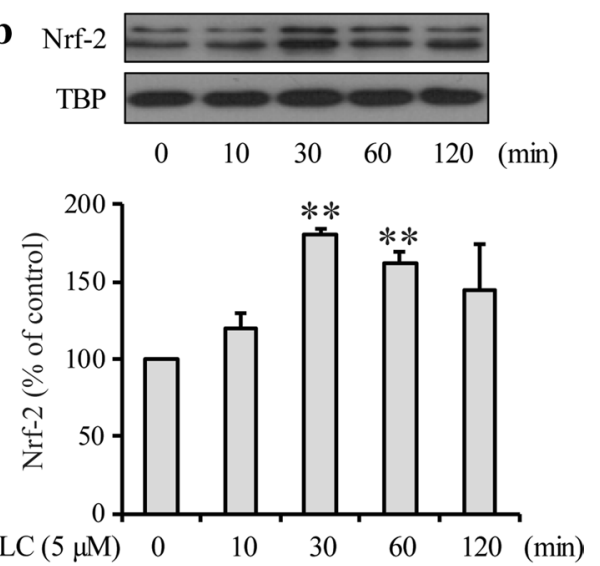

Fig. $3 \mathrm{MLC}$ increases HO-1 expression and Nrf-2 activity. a After pretreating with MLC (5 $\mu \mathrm{M})$ for 1 h, the cells were washed and further incubated in fresh media for $2,5,11$ or $23 \mathrm{~h}$. The expression of $\mathrm{HO}-1$ was determined by Western blot analysis. ${ }^{*} p<0.05$ and ${ }^{* *} p<0.01$, compared to the untreated group (0 h MLC). b After treating MLC for $10 \mathrm{~min}, 30 \mathrm{~min}, 1 \mathrm{~h}$ and $2 \mathrm{~h}$, the nuclei of HT-22 cells were fractionated from the cytosol by using NE-PER Nuclear and Cytoplasmic Extraction Reagents as described in Materials and methods. Nrf-2 proteins were detected by Western blot analysis $(n=3) .{ }^{* *} p<0.01$, compared to the untreated group (0 min MLC). The data indicate mean \pm SEM 
$191.3 \pm 21.5,695.9 \pm 33.1 \%$; 0,3 , and $6 \mathrm{~h}$ after MLC treatment, respectively). Because the translocation of Nrf-2 to the nucleus can induce the expression of $\mathrm{HO}-1$, we also measured the protein levels of Nrf-2 from the nuclear fraction of the HT-22 cells. In this experiment, MLC significantly increased the protein level of Nrf-2 with a maximal value at $30 \mathrm{~min}$ (Fig. $3 \mathrm{~b}, 0 \mathrm{~min}$ : $100 \pm 0.0,30$ min: $180.1 \pm 4.3 \%, p<0.01)$. These results suggest that MLC may induce the expression of $\mathrm{HO}-1$ by regulating $\mathrm{Nrf}-2$ translocation. For additional confirmation of HO-1 involvement in the MLC effects, SnPP, an HO-1 inhibitor, was tested in cell viability and ROS production under glutamate-induced oxidative conditions (Fig. 4). Both the MLC-increased cell viability and reduction rate of ROS were markedly affected by the addition of SnPP (Fig. 4). In the presence of $20 \mu \mathrm{M}$ SnPP, the MLC-enhanced neuronal viability was reduced to the level similar to that of cells which were not pretreated with MLC under the glutamate-induced oxidative condition (Glu only: $31.4 \pm 4.8$, Glu+MLC: $80.9 \pm 0.1$, Glu + MLC+SnPP: $45.7 \pm 6.1 \%$, $p<0.01$, compared with Glu + MLC). The effect of MLC to reduce ROS production was also partially but significantly blocked by the addition of SnPP (Glu + MLC: $104.8 \pm 2.8$, Glu + MLC+SnPP: $125.3 \pm 3.4 \%, \quad p<0.05$, compared with Glu + MLC). These results indicate that the Nrf-2/HO-1 signaling pathway may participate in the antioxidant signaling pathway that is activated by MLC.

\section{The PI3K/Akt pathway is involved in the neuroprotective effects of MLC}

The PI3K/Akt signaling pathway contributes to neuroprotective mechanisms by regulating the expression of
HO-1 [16]. In its main signaling cascade, PI3K activation mediates the phosphorylation of Akt, also known as protein kinase $\mathrm{B}$, which in turn translocates cytosolic Nrf-2 to the nucleus $[17,18]$. To evaluate the involvement of PI3K in MLC-induced $\mathrm{Nrf}-2$ accumulation in the nucleus, the phosphorylation rates of Akt were observed by Western blot analysis. As shown in Fig. 5,

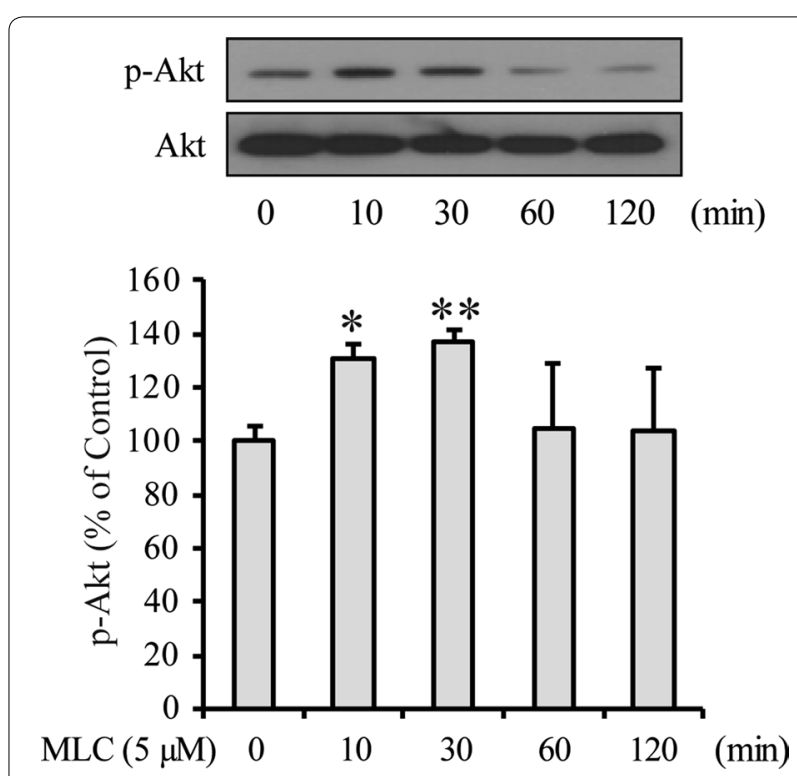

Fig. 5 MLC induces the phosphorylation of Akt. HT-22 cells were incubated with MLC for the indicated times. The cells were lysed and the $p$-Akt and Akt proteins were detected by Western blot analysis. The data indicate mean $\pm \operatorname{SEM}(n=3)$, and ${ }^{*} p<0.05$ or ${ }^{* *} p<0.01$, compared to the untreated group ( 0 min MLC)
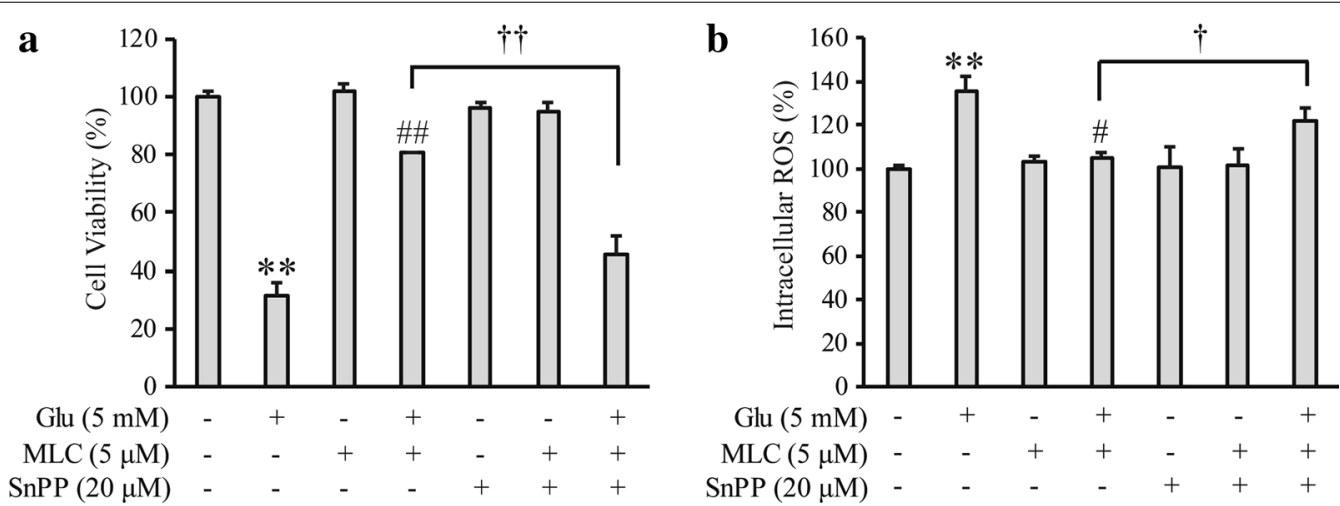

Fig. 4 The antioxidant effects of MLC are blocked by HO-1 inhibition. HT-22 cells were pretreated with SnPP ( $20 \mu \mathrm{M})$ for $2 \mathrm{~h}$ before treating with MLC. After pretreating with MLC (1, 3,5 $\mu \mathrm{M})$ for $1 \mathrm{~h}$, these cells were washed and incubated with glutamate $(5 \mathrm{mM})$ for $12 \mathrm{~h}$. a The cell viability was measured by using MTT $(2 \mathrm{mg} / \mathrm{ml})$ solution. ${ }^{* *} p<0.01$, compared to the untreated group (no drugs). ${ }^{\# \#} p<0.01$, compared to the group treated with glutamate alone. ${ }^{++} p<0.01$, indicating the significance with or without SnPP pretreatment in groups treated with MLC and glutamate. $\mathbf{b}$ Intracellular ROS levels were measured using ROS-sensitive fluorescent DCF-DA. ${ }^{* *} p<0.01$, compared to the untreated group (no drugs). ${ }^{\#} p<0.05$, compared to the group treated with glutamate alone. ${ }^{\dagger} p<0.05$, indicating the significance with or without SnPP pretreatment in groups treated with MLC and glutamate. The data indicate mean $\pm \operatorname{SEM}(n=3)$ 
the treatment with MLC significantly increased the phosphorylation of Akt at $10 \mathrm{~min}$ and $30 \mathrm{~min}$, consistent with the pattern of Nrf- 2 change induced by MLC $(100.0 \pm 5.7$, $131.1 \pm 5.2$, and $137.1 \pm 4.7 \%$; at 0,10 and $30 \mathrm{~min}$ after MLC treatment, respectively). This indicates that PI3K signaling may also contribute to the MLC-induced neuroprotective effects. PI3K involvement was additionally confirmed by testing the effect of LY294002, a PI3K inhibitor, on the antioxidant effects of MLC. In Fig. 6, LY294002 significantly reduced the protein level of HO-1 in cells pretreated with MLC (no treatment: $100.0 \pm 29.5$; Glu+MLC: $\quad 306.6 \pm 57.3 ; \quad$ Glu + MLC+LY294002:
$128.0 \pm 33.1 \%)$. MLC-increased expression of Nrf-2 was also reduced by LY294002 treatment (Fig. 2b, MLC: $134.3 \pm 6.6, \quad$ MLC $+\mathrm{LY} 294002: \quad 104.7 \pm 6.0 \%, \quad p<0.01)$. This strongly suggests that the MLC effect via activating Nrf-2 signaling may depend on PI3K pathway. Moreover, the ability of MLC to enhance neuronal viability was remarkably blocked by LY294002 treatment (Fig. 6c, Glu + MLC: $89.7 \pm 1.5$, Glu + MLC+LY294002: $75.3 \pm 1.3 \%, p<0.01$, compared to Glu + MLC). These results indicate that activation of the PI3K/Akt signaling pathway may be required for the neuroprotective effects of MLC to regulate HO-1-mediated signaling. a

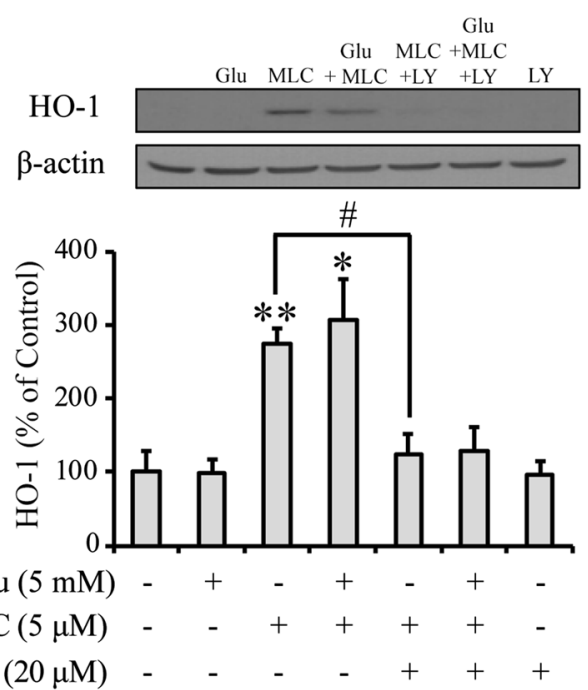

$\mathbf{b}$
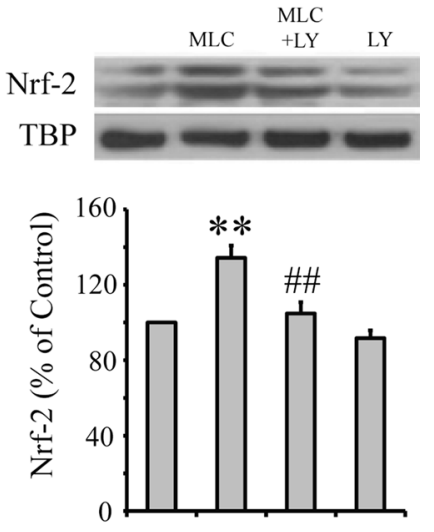

$\operatorname{MLC}(5 \mu \mathrm{M})$ LY294002 (20 $\mu \mathrm{M})$ c

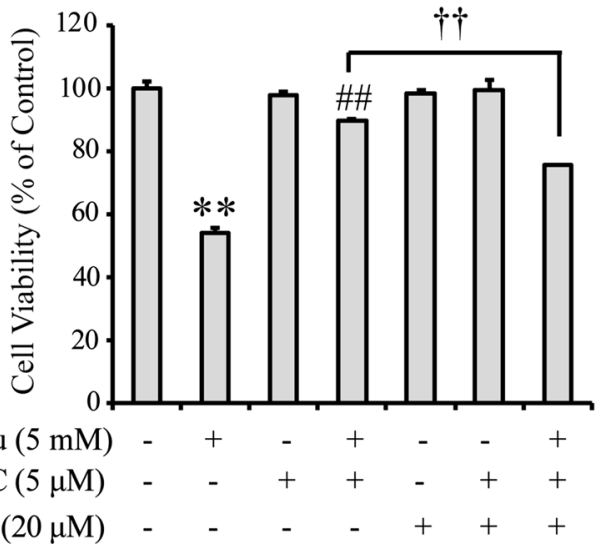

Fig. 6 MLC protects HT-22 neurons via activating PI3K/Akt pathway. HT-22 cells were pretreated with LY294002 (20 $\mu \mathrm{M})$ for $1 \mathrm{~h}$ before treating with MLC. After pretreating with MLC ( $5 \mu \mathrm{M})$ for $1 \mathrm{~h}$, these cells were washed and incubated with glutamate $(5 \mathrm{mM})$ for $5 \mathrm{~h}$ or $12 \mathrm{~h}$ for measuring HO-1 expression or cell viability, respectively. a Changes of HO-1 protein expression detected by Western blot analysis. ${ }^{*} p<0.05$ or ${ }^{* *} p<0.01$, compared to the untreated group (no drugs). ${ }^{*} p<0.05$, indicating the significant with or without LY294002 in the group treated with MLC. $\mathbf{b}$ Changes of Nrf-2 expression. ${ }^{* *} p<0.01$, compared to the untreated group (no drugs). ${ }^{\#} p<0.01$, compared to the MLC-treated group. c Changes of cell viability measured by MTT assay. ${ }^{*} p<0.01$, compared to the untreated group (no drugs), ${ }^{\# \#} p<0.01$, compared to the group treated with glutamate alone. ${ }^{+\dagger} p<0.01$, indicating the significance with or without LY294002 pretreatment in groups treated with MLC and glutamate. The data indicate mean $\pm \operatorname{SEM}(n=3)$ 


\section{Discussion}

The major finding of the present study was that MLC, a cyclopentenedione isolated from Lindera erythrocarpa Makino, had neuroprotective effects on glutamateinduced oxidative stress in HT-22 cells without microglial regulation. This was confirmed by observing the enhanced neuronal viability, reduced ROS production, increased HO-1 expression and enhanced Nrf-2 transcription in MLC-pretreated cells under glutamateinduced oxidative conditions. The antioxidant effect of MLC required the activation of PI3K/Akt signaling, as a PI3K inhibitor completely blocked the ability of MLC to increase $\mathrm{HO}-1$ expression and cell survival rates. Together with our previous report [11], these results provide evidence that MLC may enhance neuronal viability under oxidative conditions associated with neurodegenerative pathogenesis by activating neuronal antioxidant signaling, as well as microglial neuroinflammatory processing.

In mammalian nervous systems, the excessive production of intracellular ROS causes a variety of diseases by damaging cellular components, such as DNA, lipids, and proteins. In particular, ROS-induced neuronal cell death is irreversible, so finding new antioxidant agents has been the focus of studies to research neurodegenerative diseases mediated with oxidative signaling. Glutamatemediated toxicity is well known as a major cause to induce dysfunctions in many types of neurons via activating oxidative signaling [4, 19-22]. Also, HT-22 neurons are commonly used to study oxidative glutamate toxicity because they are highly responsive to extracellular glutamate, which suppresses cystine uptake via inhibiting glutamate/cystine antiporters, resulting in oxidationmediated neuronal cell death [4, 20, 23]. In this study, $5 \mathrm{mM}$ glutamate treatment was used to increase intracellular ROS production and cause cell death in HT-22 cells (Figs. 1, 2). Under this oxidative condition, the cells would have activated several antioxidant systems, including endogenous antioxidants (e.g., glutathione and bilirubin) and antioxidant enzymes (e.g., catalase, superoxide dismutase, glutathione reductase, and $\mathrm{HO}-1)$ to maintain ROS homeostasis [24]. Therefore, the MLC effects observed here may be specific for the antioxidant signaling pathways in neurons that express many types of glutamate receptors.

We previously reported that the microglia-mediated neuroprotective effects of MLC were due to the inhibition of neuronal inflammation [11]. However, it was not clear if MLC might directly protect neurons against oxidative stress without microglia regulation. In the present study, MLC certainly suppressed glutamate-induced neuronal death, even in the absence of microglia, strongly suggesting that neuronal signaling may be sufficient for MLC to protect neuronal cells from oxidative stress (Fig. 1). Even though it is not an endogenous antioxidant factor, the neuroprotective effect of MLC seems to be due to the suppression of glutamate-increased ROS production (Fig. 2). These results are also consistent with previous studies reporting that phytochemicals exerted protective effects by activating cytoprotective proteins, including HO-1 [25-27]. Furthermore, the antioxidant effects of MLC to directly regulate $\mathrm{HO}-1$ expression, as well as Nrf-2 activation, were experimentally confirmed in this study (Fig. 3). The transcription factor Nrf-2, which is normally distributed in the cytoplasm and activated by being translocated to the nucleus, actively regulates HO-1 expression by binding to ARE [6]. Taken together, our results provide evidence that the enhanced HO-1 expression by MLC may be a key factor to inhibit glutamate-induced oxidative stress.

How does MLC regulate $\mathrm{HO}-1$ expression and $\mathrm{Nrf}-2$ activation? In the previous study, it has been reported that the activation of Nrf- 2 was controlled by its upstream regulators, such as PI3K [6]. The PI3K/Akt pathway is a well-known signaling cascade that regulates cellular growth and inhibits apoptotic processing in response to extracellular signals [16]. In this pathway, PI3K has a role to phosphorylate phosphatidylinositol- $(4,5)$ bisphosphate (PIP2) to phosphatidylinositol $(3,4,5)$-trisphosphate (PIP3) and activate Akt [17]. Then, PI3K-activated Akt sequentially phosphorylates Nrf-2 to promote its translocation from the cytoplasm to the nucleus [18]. Those reports support our results showing that MLC treatment significantly increased the expression of phosphorylated Akt, as well as Nrf-2 accumulation in the nucleus (Fig. 5). The requirement of Akt phosphorylation for the MLC effects was additionally confirmed by showing that a PI3K inhibitor (LY294002) blocked the enhancement of both HO-1 and Nrf-2 expression and reduced the neuronal viability in cells pretreated with MLC (Fig. 6). These results strongly suggest that the activation of the PI3K/ Akt pathway should be preceded to activate Nrf-2 signaling which is absolutely required for antioxidant effects of MLC, consistent with previous reports $[18,28]$. However, LY294002 did not completely block the MLC effects, as shown in Fig. 6, indicating that additional functions of MLC to affect another antioxidant signaling pathway may exist in neurons. Mitogen-activated protein kinases (MAPKs) and ubiquitin-proteasomes are also known to be involved in a major pathway activating $\mathrm{Nrf} 2 / \mathrm{HO}-1$ signaling, but their involvement was not investigated in this study [29, 30].

We did not confirm any changes in Nrf-2 ubiquitination level by MLC in this study. However, some natural compounds have been reported to exhibit antioxidant effects by activating the Nrf-2 pathway [31]. Quercetin 


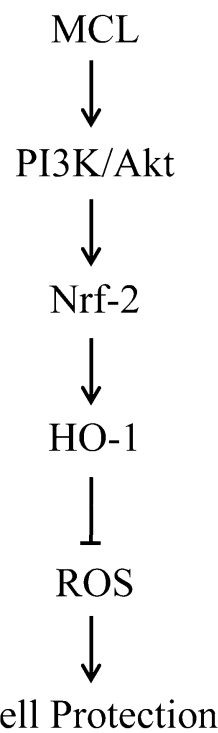

Fig. 7 Hypothetical model of the antioxidant signaling cascade for MLC-induced neuroprotection in HT-22 cells. MLC promotes the activation of PI3K/Akt pathway which phosphorylates $\mathrm{Nrf}-2$ and then induces its translocation to the nuclear. In the nucleus, Nrf2 binding to ARE promotes HO-1 transcription. Eventually, the expressed HO-1 reduces ROS and protects neurons. MLC methyl lucidone, PI3K: phosphatidylinositol 3-kinase, Nrf-2 nuclear transcription factor-E2-related factor 2, HO-1 heme oxygenase, ARE antioxidant response element, $R O S$ reactive oxygen species

has been reported to increase Nrf- 2 mRNA and protein synthesis, and sulforaphane directly isolated Nrf-2 from Keap1, inhibited degradation of Nrf-2 and then activated Nrf-2 through degradation of Keap1 [32, 33]. Similar to these compounds, MLC is thought to activate the PI3K/Akt pathway, promoting the transport of Nrf-2 into the nucleus and increasing the expression of HO-1 (Fig. 7).

\section{Authors' contributions}

JYP and KA who were equally contributed to this study, mainly performed experiments related with cell culture, protein detection and ROS signalings. YJC, JHL and JW performed and supported the additional experiment for protein detection. YSY supported data analysis and statistical evaluation. SYE supported the experimental design and supervised the current study. SCJ mainly supervised the current study as a corresponding author. All authors read and approved the final manuscript.

\section{Funding}

The work was supported by the National Research Foundation of Korea (NRF) Grant funded by the Korea government (MEST) (No. NRF-2016R1D1A1B0101086)

\section{Availability of data and materials}

The datasets used and analyzed during the current study are available from the corresponding author on reasonable request.

\section{Competing interests}

The authors declare that they have no competing interests.

\section{Author details}

${ }^{1}$ Department of Physiology, School of Medicine, Jeju National University, 102 Jejudaehakno, Jeju-si, Jeju-do 63243, Republic of Korea. ${ }^{2}$ Institute of Medical Science, Jeju National University, Jeju 63243, Republic of Korea. ${ }^{3}$ Interdisciplinary Graduate Program in Advanced Convergence Technology \& Science, Jeju National University, Jeju 63243, Republic of Korea. ${ }^{4}$ Division of Brain Diseases, Center for Biomedical Science, Korea National Institute of Health, Cheongju 28159, Republic of Korea. ${ }^{5}$ Korea Brain Research Institute, Daegu 41068, Republic of Korea. ${ }^{6}$ Neurology 1, The Second Affiliated Hospital of Xinxiang Medical University, Henan 453002, China.

Received: 13 August 2019 Accepted: 2 November 2019

Published online: 08 November 2019

\section{References}

1. Beal MF (1995) Aging, energy, and oxidative stress in neurodegenerative diseases. Ann Neurol 38(3):357-366

2. Coyle JT, Puttfarcken P (1993) Oxidative stress, glutamate, and neurodegenerative disorders. Science 262(5134):689-695

3. Jenner P (1994) Oxidative damage in neurodegenerative disease. Lancet 344(8925):796-798

4. Murphy TH, Miyamoto M, Sastre A, Schnaar RL, Coyle JT (1989) Glutamate toxicity in a neuronal cell line involves inhibition of cystine transport leading to oxidative stress. Neuron 2(6):1547-1558

5. Halliwell B (1996) Antioxidants in human health and disease. Annu Rev Nutr 16:33-50

6. Surh YJ (2003) Cancer chemoprevention with dietary phytochemicals. Nat Rev Cancer 3(10):768-780

7. Kang KW, Lee SJ, Park JW, Kim SG (2002) Phosphatidylinositol 3-kinase regulates nuclear translocation of NF-E2-related factor 2 through actin rearrangement in response to oxidative stress. Mol Pharmacol 62(5):1001-1010

8. Bryan HK, Olayanju A, Goldring CE, Park BK (2013) The Nrf2 cell defence pathway: Keap1-dependent and -independent mechanisms of regulation. Biochem Pharmacol 85(6):705-717

9. Ichino K, Tanaka H, Ito K, Tanaka T, Mizuno M (1988) Two new dihydrochalcones from Lindera erythrocarpa. J Nat Prod 51(5):915-917

10. Oh HM, Choi SK, Lee JM, Lee SK, Kim HY, Han DC et al (2005) Cyclopentenediones, inhibitors of farnesyl protein transferase and anti-tumor compounds, isolated from the fruit of Lindera erythrocarpa Makino. Bioorg Med Chem 13(22):6182-6187

11. Cui Y, Wu J, Jung SC, Kim GO, Kyeong Ko R, Lee HJ et al (2012) Neuroprotective effect of methyl lucidone against microglia-mediated neurotoxicity. Eur J Pharmacol 690(1-3):4-12

12. Breyer A, Elstner M, Gillessen T, Weiser D, Elstner E (2007) Glutamateinduced cell death in neuronal HT22 cells is attenuated by extracts from St. John's wort (Hypericum perforatum L.). Phytomedicine 14(4):250-255

13. Fu Y, Koo MW (2006) EGCG protects HT-22 cells against glutamateinduced oxidative stress. Neurotox Res 10(1):23-30

14. Le WD, Xie WJ, Appel SH (1999) Protective role of heme oxygenase-1 in oxidative stress-induced neuronal injury. J Neurosci Res 56(6):652-658

15. Chen K, Gunter K, Maines MD (2000) Neurons overexpressing heme oxygenase-1 resist oxidative stress-mediated cell death. J Neurochem 75(1):304-313

16. Hawkins PT, Anderson KE, Davidson K, Stephens LR (2006) Signalling through Class I PI3Ks in mammalian cells. Biochem Soc Trans 34(Pt 5):647-662

17. Vanhaesebroeck B, Alessi DR (2000) The PI3K-PDK1 connection: more than just a road to PKB. Biochem J. 346(Pt 3):561-576

18. Wang L, Chen Y, Sternberg P, Cai J (2008) Essential roles of the PI3Kinase/ Akt pathway in regulating Nrf2-dependent antioxidant functions in the RPE. Invest Ophthalmol Vis Sci 49(4):1671-1678

19. Oka A, Belliveau MJ, Rosenberg PA, Volpe JJ (1993) Vulnerability of oligodendroglia to glutamate: pharmacology, mechanisms, and prevention. J Neurosci 13(4):1441-1453

20. Davis JB, Maher P (1994) Protein kinase C activation inhibits glutamateinduced cytotoxicity in a neuronal cell line. Brain Res 652(1):169-173 
21. Miyamoto M, Murphy TH, Schnaar RL, Coyle JT (1989) Antioxidants protect against glutamate-induced cytotoxicity in a neuronal cell line. J Pharmacol Exp Ther 250(3):1132-1140

22. Vornov JJ, Coyle JT (1991) Glutamate neurotoxicity and the inhibition of protein synthesis in the hippocampal slice. J Neurochem 56(3):996-1006

23. Maher P, Davis JB (1996) The role of monoamine metabolism in oxidative glutamate toxicity. J Neurosci 16(20):6394-6401

24. Blokhina O, Virolainen E, Fagerstedt KV (2003) Antioxidants, oxidative damage and oxygen deprivation stress: a review. Ann Bot 91:179-194

25. Hwang YP, Jeong HG (2008) The coffee diterpene kahweol induces heme oxygenase-1 via the PI3K and p38/Nrf2 pathway to protect human dopaminergic neurons from 6-hydroxydopamine-derived oxidative stress. FEBS Lett 582(17):2655-2662

26. Senthil Kumar KJ, Liao JW, Xiao JH, Gokila Vani M, Wang SY (2012) Hepatoprotective effect of lucidone against alcohol-induced oxidative stress in human hepatic HepG2 cells through the up-regulation of HO-1/Nrf-2 antioxidant genes. Toxicol In Vitro 26(5):700-708

27. Zhang Z, Cui W, Li G, Yuan S, Xu D, Hoi MP et al (2012) Baicalein protects against 6-OHDA-induced neurotoxicity through activation of Keap1/ Nrf2/HO-1 and involving PKCalpha and PI3K/AKT signaling pathways. J Agric Food Chem 60(33):8171-8182

28. Lee JM, Hanson JM, Chu WA, Johnson JA (2001) Phosphatidylinositol 3-kinase, not extracellular signal-regulated kinase, regulates activation of the antioxidant-responsive element in IMR-32 human neuroblastoma cells. J Biol Chem 276(23):20011-20016

29. Xu C, Yuan X, Pan Z, Shen G, Kim JH, Yu S et al (2006) Mechanism of action of isothiocyanates: the induction of ARE-regulated genes is associated with activation of ERK and JNK and the phosphorylation and nuclear translocation of Nrf2. Mol Cancer Ther 5(8):1918-1926

30. Li H, Wu S, Shi N, Lian S, Lin W (2011) Nrf2/HO-1 pathway activation by manganese is associated with reactive oxygen species and ubiquitinproteasome pathway, not MAPKs signaling. J Appl Toxicol 31(7):690-697

31. Wu KC, McDonald PR, Liu J, Klaassen CD (2014) Screening of natural compounds as activators of the keap1-nrf2 pathway. Planta Med 80(1):97-104

32. Tanigawa S, Fujii M, Hou DX (2007) Action of Nrf2 and Keap1 in ARE-mediated NQO1 expression by quercetin. Free Radical Biol Med 42(11):1690-1703

33. Zhang DD, Lo SC, Sun Z, Habib GM, Lieberman MW, Hannink M (2005) Ubiquitination of Keap1, a BTB-Kelch substrate adaptor protein for Cul3, targets Keap1 for degradation by a proteasome-independent pathway. J Biol Chem 280(34):30091-30099

\section{Publisher's Note}

Springer Nature remains neutral with regard to jurisdictional claims in published maps and institutional affiliations.

\section{Submit your manuscript to a SpringerOpen ${ }^{\circ}$ journal and benefit from:}

- Convenient online submission

Rigorous peer review

- Open access: articles freely available online

- High visibility within the field

- Retaining the copyright to your article

Submit your next manuscript at springeropen.com 